\title{
Awareness, facilitators and barriers to policy implementation related to obesity prevention for primary school children in Malaysia \\ ${ }^{1}$ Camelina Chan, ${ }^{2}$ Foong Ming Moy, ${ }^{3}$ Jennifer NW Lim, ${ }^{1}$ Maznah Dahlui \\ ${ }^{1}$ Center for Population Health, Department of Social and Preventive Medicine, 6 Faculty of Medicine, University of Malaya, Kuala Lumpur, Malaysia
}

${ }^{2}$ Julius Centre University of Malaya, Department of Social \& Preventive Medicine, 8 Faculty of Medicine, University of Malaya, Kuala Lumpur, Malaysia.

${ }^{3}$ Faculty of Education, Health and Wellbeing, University of Wolverhampton, United Kingdom

Address correspondence to:

Camelina Chan

Center for Population Health

Department of Social and Preventive Medicine, Faculty of Medicine, University of Malaya, 50603, Kuala Lumpur, Malaysia

Email : camelinachan@gmail.com

Tel : +603 79674756

Fax No: +603 79674975

Co-Authors

Assoc. Prof. Dr. Foong Ming Moy

Julius Centre University of Malaya

Department of Social and Preventive Medicine, Faculty of Medicine, University of Malaya, 50603, Kuala Lumpur, Malaysia

Email: moyfm@ummc.edu.my

Dr. Jennifer NW Lim

Institute of Public Health, Social Work and Care

Faculty of Education, Health and Wellbeing

University of Wolverhampton

Wulfruna Street

Wolverhampton

WV1 1LY

United Kingdom

Email: jennifernw.lim@wlv.ac.uk 
Prof. Dr. Maznah Dahlui

41 Center for Population Health

42 Department of Social and Preventive Medicine,

43 Faculty of Medicine,University of Malaya,

$44 \quad$ 50603, Kuala Lumpur, Malaysia

$45 \quad$ Email: maznahd@ummc.edu.my

46

47

48

49

50

51

52

53

54

55

56

57

58

59

60

61

62

63

64

65

66

67

68

69

70

71

72

73

74

75

76

77

78

79 


\section{ABSTRACT}

81 Purpose. To assess the awareness, facilitators and barriers to policy implementation related to obesity prevention for primary school children.

83 Design. A cross-sectional study administered using an online questionnaire.

84 Setting. Conducted in 447 primary schools in a state in Malaysia.

85 Subjects. One school administrator from each school served as a subject.

86 Measures. The questionnaires consisted of 32 items on awareness, policy

87 implementation; facilitators and barriers to policy implementation.

88 Analysis. Descriptive analysis was used to describe the awareness, facilitators and

89 barriers of policies implementation. Association between schools' characteristics and

90 policy implementation was assessed using logistic regression.

91 Results. The majority (90\%) of school administrators were aware of the policies.

92 However, only $50 \%$ to $70 \%$ of schools had implemented the policies fully. Reported

93 barriers were lack of equipment, insufficient training and limited time to complete

94 implementation. Facilitators of policy implementation were commitment from the

95 schools, staff members, students and canteen operators. Policy implementation was 96 comparable in all school types and locality; except the policy on" Food and Drinks sold

97 at the school canteens" was implemented by more rural compared to urban schools

98 (Odds ratio 1.74, 95\% Confidence Interval: 1.13, 2.69).

99 Conclusion. Majority of the school administrators were aware of the existing policies, 100 however, the implementation was only satisfactory. The identified barriers to policy 101 implementation were modifiable and thus the stakeholders should consider re102 strategizing plans in overcoming them. 
103

104 Keywords. school policies, school guidelines, facilitators, barriers, obesity, school 105 children

106

107

108

109

110

111

112

113

114

115

116

117

118

119 


\section{INTRODUCTION}

121 Overweight and obesity is a major public health problem in our country, Malaysia.

122 In 2010, the prevalence of adult obesity in our country was ranked the first in South-

123 East Asia and sixth in Asia ${ }^{1-3}$. There was a sevenfold increase in obesity from 1996

$124(4.4 \%)^{4}$ to $\left.2015(33.4 \%)\right)^{4,5}$ The prevalence of childhood obesity (BMI for age >+2SD)

125 in 2015 was $11.9 \%$ (CI: 10.9-12.9)..$^{5}$ This trend is worrisome since Malaysia has a

126 young age populations of $26 \%{ }^{6}$ and obesity is found to persist from childhood into

127 adulthood.

128

129 In many countries, the rising prevalence of childhood obesity was recognized by

130 their governments resulting in various initiatives and interventions implemented to

131 promote healthy behavior among school children. Many national policies were

132 specifically developed for school children, which had positive effects on their diet (e.g.

133 the free flow of fresh fruits and vegetables in the school) ${ }^{7}$ and physical activities. ${ }^{8,9}$

134

135 In view of the rising prevalence of obesity in our country, managing obesity among

136 school children has been given a priority by the Ministry of Health. ${ }^{10}$ Many programs

137 were introduced to implement various policies for school children such as the

138 "Integrated School Health Program", "Self-evaluation Program" and "School Health

139 Promotion-Young Doctors Program. ${ }^{11-16}$ The Integrated School Health Program is

140 implemented by the Ministry of Education in collaboration with the Ministry of Health

141 to provide health services (such as the school health service, school dental service, and

142 school environmental health service) to the school children. Self-evaluation program is 
143 part of the initiatives under the implementation of Management of Healthy School

144 Canteens guidelines to ensure that school canteen operators (who sell food and beverage

145 at school canteens) provide safe and healthy meals for school children. The School

146 Health Promotion-Young Doctors Program is part of health promotion initiatives in the

147 school. Peer mentors were selected among the school children of Year 4, 5 and 6 as

148 change agents in the schools to improve the students' knowledge and skills. These

149 "young doctors" will try to promote positive health behaviour among their own peers,

150 school communities and family members.

151

152 The effectiveness of school-based policies (either written directives, documented 153 programs, written guidelines) against childhood obesity depends on their 154 implementation. Often, implementation is not optimal even when the policies are 155 publicly mandated. ${ }^{17}$ Therefore, evaluation of policy implementation is essential. To 156 date, only few studies have examined facilitators and barriers influencing the 157 implementation of school-based obesity prevention policies. ${ }^{17}$ Therefore, the purpose of 158 this study was to assess the awareness of the available related policies on obesity 159 prevention for school children among the school administrators, the implementation 160 status, and factors influencing its implementation.

161

162 METHODS

163 Policies are referred to written directives, guidelines, manuals and programs related to 164 obesity prevention. ${ }^{18}$ 
168 In-depth interviews (IDI) with key personnel were conducted by the principal 169 investigator to identify the available policies related to obesity prevention for school 170 children as well as to understand its process of implementation in Malaysia. The 171 interviews were conducted from May to August 2014. The respondents were two 172 policy makers from the Non-Communicable Disease division, Ministry of Health 173 headquarters; seven school health team members (i.e. four medical doctors, two 174 nutritionist and one school nurse) at district level and six school administrators from 175 Malay-medium national primary schools $(\mathrm{SK})(\mathrm{n}=2)$ and non-Malay-medium primary 176 schools (also known as vernacular schools) ( $\mathrm{n}=4$, two from SJKT and two SJKC 177 respectively). The vernacular schools included the Tamil-medium national school 178 (SJKT) and Chinese-medium national school (SJKC). The interviews were conducted 179 at the interviewees' premises to allow the interviewer to observe the actual environment 180 and better understand any specific issues when they were raised.

181

182 Purposive sampling method was used in identifying and selecting participants for the 183 IDIs. The IDIs were conducted using the guidelines designed by the research team. The 184 guidelines consisted of 14 open-ended items with probing questions for a systematic 185 interview (refer to appendix). The items included understanding the policies that are 186 currently being implemented in primary schools, how policies were implemented and 187 what were the factors that might facilitate or act as barriers to the implementation. 188 Interviews were conducted until content saturation was achieved (when similar 
189 comments were consistently repeated). Oral informed consents were given by the 190 participants for permission to record all interviews. All tape recordings were transcribed

191 verbatim. The transcriptions were imported into Nvivo7 for coding and analysis.

192 Finally, the findings from the IDIs were used to design and develop an online

193 questionnaire using the Google application. The created Google online questionnaire

194 enabled us to compile the survey responses into the Excel spreadsheet for analysis.

195

196 The questionnaire included 32 items covering the topics on awareness,

197 implementation process and factors that impeded or facilitated the implementation of

198 the policy or guidelines. Awareness was measured by the responses of 'yes, no, not

199 sure". The questions on implementation focused on items considered relevant to

200 predicting the extent to which the school complied with the existing policies; while the

201 questions on factors that facilitated or acted as barriers were based on the challenges

202 faced in implementing the policies. The internal consistency (Cronbach alpha) of the

203 questionnaire was 0.80 .

204

205 The questionnaire was written in the national (Malay) language and was validated by

206 three experts in the similar field of study for face and content validation. Pre-testing

207 was carried out among 11 school administrators who were not involved in the study.

209 Study design and Sampling Methods

210 This was a cross-sectional study conducted from October 2014 to February 2015

211 involving all primary schools (attended by children age 7-12 years old) in the state of 
212 Selangor Darul Ehsan. Universal sampling was carried out where all primary schools in

213 the urban and rural areas of Selangor $(n=641)$ were invited to participate.

214

\section{Data collection}

216 A self-administered online questionnaire was used to assess the respondents' 217 awareness on the existing policies related to obesity prevention among school children.

218 All primary school administrators in Selangor were invited to participate in the study

219 via emails and further followed up using official letters and telephone communication.

220 After agreement to participation was obtained, the school administrators

221 (headmaster/assistance headmaster/physical education teacher) were briefed (via

222 telephone communication) about the purpose of the research. Upon receiving verbal 223 informed consent and verification of the schools' email addresses, the questionnaire was

224 emailed to the schools. The researcher made follow-up calls every two weeks to ensure

225 the school administrators completed and returned the questionnaires.

\section{Statistical Analysis}

228 All compiled data in the Excel spreadsheet were exported into Statistical Package for 229 the Social Sciences (SPSS) for Windows, version 22. Descriptive statistics were used 230 to describe the awareness and factors that facilitated or acted as barriers to policy 231 implementation. Logistic regression was used to examine the association between 232 schools' characteristics and policy implementation. The significant level was preset 233 at $\mathrm{p}<0.05$. 


\section{Ethics clearance}

236 Ethics clearance (Reference No: 989.27) was obtained from the University of Malaya

237 Medical Centre Research Ethics Committee. Permission to conduct research at the 238 schools was obtained from the Ministry of Education (Reference No: $239 \mathrm{KP}(\mathrm{BPPDP}) 603 / 5 / \mathrm{JLD} .13$ (234). Informed consent was received from all the 240 respondents.

\section{RESULTS}

The online survey was completed by 447 out of 641 school administrators, giving a

244 response rate of $70 \%$. Each school was represented by one respondent. Among the 245 respondents, $56.6 \%$ were assistant headmasters, $36.9 \%$ were headmasters and $6.5 \%$ 246 were physical education teachers. Among all the schools, 67.6\%, 17.2\%, and 15.2\%

247 were SK, SJKC and SJKT, respectively. Based on geographical location, rural and 248 urban schools were $42.3 \%$ and $57.7 \%$, respectively. There was no difference in school 249 type and geographical location between the responded and non-responded schools.

Awareness and Status of Policy Implementation

252 The existing policies related to obesity prevention among school children were mainly 253 developed by the Ministry of Education in collaboration with the Ministry of Health and 254 local councils (Table 1). All national policies that were developed for the Ministry of 255 Education were informed to all the government schools through circulars by their 256 respective state education offices. Implementation refers to the execution of policies.

257 There were six steps in the policy implementation identified during the IDIs. The 
258 implementation of policies depends on the resources needed and availability of 259 resources at schools. The timing of policy implementation varied. Some were 260 implemented as soon as directives were given, such as healthy school canteen

261 guidelines implemented by the canteen operators. However, some were implemented in

262 the next academic year, such as "1 Sport 1 Student" when the school children were

263 involved as there might be no vacant slots in the current school calendar. Monitoring of 264 policy implementation was carried out by the stakeholders through annual meetings and 265 written reports.

266 [Insert Table 1]

268 It is important for the implementers to be aware and get familiarized with the school269 based-policies before any implementation process can be optimized. Figure 1 shows the 270 level of awareness and status of full implementation of individual policies (P1 to P5) 271 related to obesity prevention for school children. The majority of the respondents (96\%) 272 reported a good awareness and familiarity with the existing policies. However, the 273 status of policy implementation was only acceptable, with P3 found to be highest (72\%) 274 followed by P1 (64.7\%), P5 (56.6\%), P2 (55.3\%) and P4 (51\%).

275 [Insert Figure 1]

277 There was no difference in the status of policy implementation according to the types of 278 schools (Table 2). Similarly, the rural and urban schools also showed no difference in 279 the implementation of all policies, except for the Guidelines for Food and Drinks Sold 
280 at the School Canteen (P3). Rural schools had higher odds of implementing the

281 Guidelines for Food and Drinks Sold at the School Canteen (P3) than urban schools

282 (OR: $1.74,95 \%$ CI: 1.13, 2.69).

283 [Insert Table 2]

\section{Facilitators and Barriers}

286 Table 3 shows the factors that facilitated or acted as the barriers to the

287 implementation of the existing policies. Most of the school administrators reported that 288 policy implementation was a priority $(82.8 \%)$ and they had taken responsibility to 289 implement the policies $(71.1 \%)$. They also reported familiarity and had sufficient 290 knowledge in the implementation process (60.6\%). Overall, the school administrators 291 had received good support from their staff $(86.6 \%)$ and school canteen operators 292 (81.7\%) in policy implementation. They also received co-operation from the majority of 293 their students $(77.2 \%)$. Furthermore, $51.2 \%$ of the school administrators reported that 294 their schools had sufficient funding to carry out the given mandate. Factors that acted as 295 barriers to the implementation of the existing policies were lack of equipment (78.5\%), 296 followed by having limited time $(71.8 \%)$, insufficient training $(71.6 \%)$, no penalty 297 given for non-compliance $(61.1 \%)$, too much paperwork $(60 \%)$ and lack of support 298 from parents and community (51.7\%).

299 [Insert Table 3]

300

DISCUSSION 
We found that majority of the school administrators were familiar and understood the implementation process of the existing policies. However, only two policies; Policy 1 student 1 sport (P1) and Guidelines of Food and Beverage sold at the school canteen

305 (P3) were implemented by more than $60 \%$ of the schools, which could be considered as 306 satisfactory. It is difficult to get a hundred percent implementation of any policy. Durlak

307 and colleague (2008) suggested that a perfect or near perfect policy implementation was unrealistic. Positive results to program adaptation have often been obtained with implementation levels around $60 \%$ to $80 \%$, while no study has documented a $100 \%$

310 implementation. ${ }^{19}$ In our study, the implementation of other policies (P2, P4, and P5)

311 was slightly lower than $60 \%$. The implementation of the Guidelines in weight 312 management of school children (P2) involved measurement of students' BMI twice a 313 year. However, issues with lack of equipment (weighing scale and stadiometer) and 314 limited time had made its implementation difficult in some schools. In addition, the 315 banning of the sales of food and beverages by mobile vendors outside the school 316 perimeters $(\mathrm{P} 4)$ needs the involvement and reinforcement of the local councils, while 317 the School Health Promotion-Young Doctors Program (P5) was made optional for 318 implementation by the authority.

320 We also found no difference in the status of implementation according to the location 321 of schools, except for the policy on 'Guidelines of Food and Beverage sold at the school 322 canteen" (P3). These guidelines aimed to guide the canteen operators and school 323 administrators on the list of foods and beverages that are permitted, not encouraged and 324 prohibited at the school canteens, and to provide information on the amount of energy in 
325 food by displaying the total calories, as well as to highlight the methods of monitoring 326 on selling food and drinks at the school canteens. ${ }^{16}$ These guidelines had been

327 incorporated into the Healthy School Canteen Program and were distributed to all the 328 schools in Malaysia. The canteen operators found the guidelines useful in preparing 329 healthy meals for the school children. However, the foods and beverages recommended 330 in the guidelines were not popular among the school children as these food and 331 beverages tended to be lower in fat, sugar, and sodium. Therefore, canteen operators 332 would tend to sell foods and beverages preferred by the school children, such as fast 333 foods. ${ }^{20}$ The compliance to this policy (P3) was better at the rural compared to the urban 334 schools since urban schools are usually located near the commercial areas which 335 provide easy access to unhealthy food and snacks. With the high competition from the 336 surrounding area and high demands from the school children, the urban school canteen 337 operators may have opted to sell foods and beverages preferred by the school children. 338 Although healthy food choices and eating behavior are incorporated in the school co339 curriculum, the school authorities should reinforce its importance. In addition, it would 340 be good if the government could subsidize healthy foods which are usually more expensive so that the canteen operators are ensured with better income. Furthermore,

342 the schools are encouraged to ease the financial burden of the canteen operators by 343 waiving or reducing the canteen premise rental or subsidize the price of healthy food 344 choices such as whole-meal bread, fruits, vegetables and dairy products.

346 In this study, the co-operation from staff and school canteen operators in policy 347 implementation was found to be above $80 \%$. However, there was less support from the 
348 parents and the community. Inter-sectoral cooperation is one of the key factors in 349 successful policy implementation ${ }^{21}$. Having parents to be actively involved would 350 improve policy implementation. Parents need to be empowered through the Parents-

351 Teachers Association that should promote and conduct school activities and programs 352 related to healthy lifestyle. Although the school administrators showed their full 353 commitment and had sufficient knowledge on policy implementation, they still need 354 additional support in providing the facilities/equipment, as well as provide sufficient 355 training to their staff. Moreover, the school administrators reported that the 356 implementation of policies involved too much paperwork which increased their work 357 burden in addition to the workload from their academic commitment. Similar results

358 have also been reported by other studies which assessed the facilitators (e.g. leadership 359 and effective communication) ${ }^{22}$ and barriers (e.g. parental support) ${ }^{23}$ of policy 360 implementation.

362 We did not find funding to be a barrier to policy implementation. Most of the 363 policies, except for $\mathrm{P} 2$, did not require financial assistance for implementation. 364 Furthermore, most of the programs were supposed to be implemented by the school canteen operators. Identified factors that acted as barriers were modifiable and thus 366 present clear opportunities for improvement in the implementation of policies. The issue 367 on additional administrative work given to the teachers and time taken by them in 368 recording the measurements of weight and height which then need to be submitted to 369 the ministry could avoid duplication if the data could be directly keyed-in to an online 370 system using a portable gadget. The issue of lacking in facilities and equipment raised 
371 by the school administrators could be due to the poor maintenance of those facilities,

372 which may be addressed by outsourcing its maintenance service. These

373 recommendations could be considered by the policymakers in re-strategizing future

374 planning.

375

376 Strengths and Limitations

377 This is the first study that assessed the implementation of related policies in Malaysia. It

378 involved multiple stakeholders such as policymakers, school health team members, and

379 school administrators which their views were included. On the other hand, this was a

380 cross-sectional study, based upon perceptions of the respondents. In addition, we did

381 not assess whether awareness and implementation of policies made a difference in

382 children's dietary and physical activity behaviors as well as the prevalence of obesity.

383 However, our findings will assist researchers and policy makers in conducting further

384 studies in improving the implementation of the current related policies and guidelines.

385 Although only public schools from one state were studied, these schools were

386 representative of schools from other states as the set-up of all the public schools in

387 Malaysia is similar.

388

389 Conclusion

390 The majority of the school administrators were aware of the existing policies related to

391 obesity prevention among primary school children; however, the implementation was

392 only satisfactory. The identified barriers to policy implementation were modifiable and

393 thus the stakeholders should consider re-strategizing plans in overcoming them. 


\section{SO WHAT? Implications for Health Promotion Practitioners and Researchers}

\section{What is already known on this topic?}

399 The effectiveness of school-based policies against childhood obesity depends on their 400 implementation. Therefore, evaluation of policy implementation is important. However, 401 to date, only a few studies have examined the factors that facilitated or acted as barriers 402 to the implementation of existing policies related to obesity prevention among school 403 children.

404

405 What does this article add?

406 This study assessed and examined the facilitators/barriers to the implementation of 407 existing policies related to obesity prevention among school children in primary schools 408 in Malaysia.

409

410 What are the implications for health promotion practice and research?

411 Our study found that some of the identified factors were modifiable and thus present

412 clear opportunities for improvement in the implementation stage.

413

414

415

416 
1. World Health Organization. Fact Sheets -Obesity and Overweight. 2014; http://www.who.int/mediacentre/factsheets/fs311/en/\#, 2014.

2. WHO. Global Health Observatory Data Repository. 2015.

3. Overweight and Obesity 2014. http://vizhub.healthdata.org/obesity/. Accessed June 142016.

4. Institute for Public Health MoHM. The Third National Health and Morbidity Survey (NHMS III) 2006. Ministry of Health, Malaysia: Institute for Public Health; 2008.

429

430

431

7. Scuderi A, Sturiale L, Timpanaro G. The Approach To The Consumption of

5. Institute for Public Health MoHM. The Fifth National Health and Morbidity Survey 2015 (NHMS 2015). Vol. II: Non-Communicable Diseases, Risk Factors \& Other Health Problems 2015.

6. Malaysia Department of Statistics. Population and Demography. 2014; https://www.statistics.gov.my/index.php?r=column/ctwoByCat\&parent_id=115 \&menu_id=L0pheU43NWJwRWVSZklWdzQ4TlhUUT09.

9. Mori N, Armada F, Willcox DC. Walking to school in Japan and childhood obesity prevention: new lessons from an old policy. American journal of public health. 2012;102(11):2068-2073.

10. Sabramani VAL, Idris, I. B., Sutan, R., Isa, Z. M., Buang, S. N., \& Ghazi, H. F. . 
11. Malaysia Ministry of Health, Malaysia Ministry of Education, Kelab Doktor Muda. Health Advisors Curriculum Guidelines Peer - Young Doctors Club. Bahagian Pendidikan Kesihatan KKM dengan kerjasama Bahagian sekolah KPM; 2008.

12. Malaysia Ministry of Health, Malaysia Ministry of Education, Kelab Doktor Muda. Curriculum Guidelines Young Doctors Club. Bahagian Pendidikan Kesihatan KKM dengan Kerjasama Bahagian Sekolah KPM 2008.

13. Malaysia Ministry of Health, Malaysia Ministry of Education, Kelab Doktor Muda. Training modules Young Doctors Club for Primary School. Third Edition ed: Bahagian Pendidikan Kesihatan dengan kerjasama Kementerian Kesihatan Malaysia; 2008.

14. Nestlé Joins Hands with Ministry of Education Encouraging Children to Adopt Healthy Lifestyles [press release]. Nestle Website: Nestle, March 3, 20152015.

15. Malaysia Ministry of Education. Policy Implementation Guide One Student One Sport. In: Education Mo, ed: Bahagian Sukan; 2011.

16. Malaysia Ministry of Education. Healthy Eating in School Implementation Guidelines. 2012.

17. Masse LC, Naiman D, Naylor PJ. From policy to practice: implementation of physical activity and food policies in schools. The international journal of behavioral nutrition and physical activity. 2013;10:71.

18. WebFinance I. Businessdictionary.com http://www.businessdictionary.com/definition/policies-and-procedures.html.

19. Durlak JA, DuPre EP. Implementation matters: a review of research on the influence of implementation on program outcomes and the factors affecting implementation. Am. J. Community Psychol. 2008;41(3-4):327-350.

20. Sharif Ishak SI, Shohaimi S, Kandiah M. Assessing the children's views on foods and consumption of selected food groups: outcome from focus group approach. Nutrition research and practice. 2013;7(2):132-138.

21. Phaitrakoon J, Powwattana A, Lagampan S, Klaewkla J. The diamond level health promoting schools (DLHPS) program for reduced child obesity in Thailand: lessons learned from interviews and focus groups. Asia Pacific journal of clinical nutrition. 2014;23(2):293-300. 
479

480

481

482

483

484

485
22. Fagen MC, Asada Y, Welch S, et al. Policy, Systems, and Environmentally Oriented School-Based Obesity Prevention: Opportunities and Challenges. Journal of prevention \& intervention in the community. 2014;42(2):95-111.

23. Clarke J, Fletcher B, Lancashire E, Pallan M, Adab P. The views of stakeholders on the role of the primary school in preventing childhood obesity: a qualitative systematic review. Obesity reviews : an official journal of the International Association for the Study of Obesity. 2013;14(12):975-988. 